\title{
LOCALLY-TRIVIAL BUNDLES AND MICROBUNDLES WITH INFINITE-DIMENSIONAL FIBERS
}

\author{
T. A. CHAPMAN ${ }^{1}$
}

\begin{abstract}
The object of this note is to establish trivialization theorems for locally-trivial bundles and microbundles in which the fiber is any one of a large class of infinite-dimensional topological vector spaces and the base space is any paracompact space. These theorems generalize results of Henderson and Wong.
\end{abstract}

1. Introduction. In this paper we prove that certain locally-trivial bundles and microbundles with infinite-dimensional fibers are trivial. Our first result concerns the triviality of locally-trivial bundles whose fibers are certain infinite-dimensional topological vector spaces (TVS's).

THEOREM 1. Let $\xi$ be a locally-trivial bundle with fiber $F$ and base $B$ any paracompact space. If $F$ is any $T V S$ which is homeomorphic $(\cong)$ to its own countable-infinite product $F^{\omega}$, then $\xi$ is trivial.

This is clearly false for finite-dimensional fibers (i.e. Euclidean spaces) and it is the product structure of $F^{\omega}$ that makes this result possible. We remark that there are many examples of infinite-dimensional TVS's $F$ which satisfy the condition $F \cong F^{\omega}$; for example any separable infinitedimensional Fréchet space [1], any infinite-dimensional Hilbert space [2], or any infinite-dimensional reflexive Banach space [2]. In fact there is no known example of an infinite-dimensional Fréchet space $F$ for which the condition $F \cong F^{\omega}$ is not satisfied.

A special case of Theorem 1 was established by Wong [9] in which $F=l_{2}$ (separable infinite-dimensional Hilbert space) and $B$ is any countable locally-finite simplicial complex. The proof we give of Theorem 1 uses techniques which are completely different from those of Wong.

In our second result we establish a version of Theorem 1 for microbundles.

Received by the editors January 3, 1972.

AMS (MOS) subject classifications (1970). Primary 55F10; Secondary 55F60.

Key words and phrases. Locally-trivial bundles, microbundles, infinite-dimensional topological vector spaces.

${ }^{1}$ Supported in part by National Science Foundation grant GP-28374. 
THEOREM 2. Let $\xi$ be a microbundle with fiber $F$ and base $B$ any paracompact space. Then $\xi$ is trivial provided that $F$ is a metric TVS which satisfies the condition $F \cong F^{\omega}$.

In [4] Henderson proved Theorem 2 with the same conditions on the fiber $F$ but with more restrictive conditions on the base $B$; namely that $B$ is a paracompact space which has the homotopy type of a simplicial (or $\mathrm{CW}$ ) complex. This result was one of the crucial steps in proving the open embedding theorem of [6] (and various other results about infinitedimensional manifolds).

The proof we give of Theorem 2 is completely independent of the microbundle techniques used by Henderson in [4]. Our idea (for both Theorems 1 and 2) is to make use of a parametric version of Wong's coordinateswitching technique which was used to prove that homeomorphisms on certain infinite-dimensional spaces are isotopic to the identity [8]. This approach enables us to completely ignore the homeomorphism group of the fiber, which is particularly necessary when the fiber is an infinitedimensional TVS (as the compact-open topology is not even jointly continuous).

2. Lemmas for Theorem 1. We first introduce some notation. For finite products $\prod_{i=1}^{n} X_{i}$ we will use $p_{i}$ to denote projection onto $X_{i}$, for $1 \leqq i \leqq n$. If each $X_{i}$ is itself a finite product, then $p_{j} \circ p_{i}$ will denote the projection of $\prod_{i=1}^{n} X_{i}$ onto the $j$ th factor of $X_{i}$. If we have functions $f_{i}: X_{i} \rightarrow Y_{i}$, for $1 \leqq i \leqq n$, then $f_{1} \times \cdots \times f_{n}$ will denote the function from $\prod_{i=1}^{n} X_{i}$ to $\prod_{i=1}^{n} Y_{i}$ defined by $f_{i} \circ p_{i}=p_{i} \circ\left(f_{1} \times \cdots \times f_{n}\right)$. If $g_{i}: X \rightarrow Y_{i}$ is a function, for $1 \leqq i \leqq n$, then $\left(g_{1}, \cdots, g_{n}\right)$ will denote the function from $X$ to $\prod_{i=1}^{n} Y_{i}$ defined by $g_{i}=p_{i} \circ\left(g_{1}, \cdots, g_{n}\right)$. The above definitions have obvious analogues for countable products.

For spaces $X, Y$, and $Z$ we say that a function $f: X \times Y \rightarrow X \times Z$ is $X$ preserving provided that $p_{1} \circ f=p_{1}$. For each $x \in X$ we let $f_{x}$ denote the function from $Y$ to $Z$ defined by $f_{x}(y)=f(x, y)$, for all $y \in Y$. We also make analogous definitions of $X$-preserving functions $f: Y \times X \rightarrow Z \times X$. We use $I$ to denote the closed interval $[0,1]$ and id $\mathrm{i}_{X}$ to denote the identity function of $X$ (where the subscript is suppressed when there is no ambiguity). We also use the term map to mean a continuous function.

The basic notion of this section is the following: A space $X$ has the reflective isotopy property if there exists an $I$-preserving homeomorphism $f$ of $X^{\omega} \times I$ onto itself such that $f_{0}=\mathrm{id}$ and $f_{1}$ interchanges the first and second coordinates, i.e. $p_{1} \circ f_{1}=p_{2}, p_{2} \circ f_{1}=p_{1}$, and $p_{i} \circ f_{1}=p_{i}$, for all $i \geqq 3$. The following lemma is due to J. E. West [7] and it identifies some spaces which have the reflective isotopy property.

LEMMA 2.1 [7, p. 579]. Every TVS has the reflective isotopy property. 
Moreover the I-preserving homeomorphism of Lemma 2.1 which switches coordinates can be required to fix the origin at each level. That is for a TVS $F$ there is an $I$-preserving homeomorphism $f$ of $F^{\omega} \times I$ onto itself such that $f_{0}=\mathrm{id}, f_{1}$ interchanges the first and second coordinates, and $f_{t}(0,0, \cdots)=(0,0, \cdots)$, for all $t \in I$. We now apply Lemma 2.1 to obtain the following result.

LEMMA 2.2. If $F$ is a TVS, then there exists an I-preserving map $\varphi: F^{\infty} \times$ $F^{\omega} \times I \rightarrow F^{\omega} \times I$ such that

(1) $\varphi \mid F^{\omega} \times F^{\omega} \times(0,1)$ is a homeomorphism of $F^{\omega} \times F^{\omega} \times(0,1)$ onto $F^{\omega} \times$ $(0,1)$

(2) $\varphi_{0}=p_{1}$ (projection of $F^{\omega} \times F^{\omega}$ on to its first factor $F^{\omega}$ ),

(3) $\varphi_{1}=p_{2}$,

(4) $\varphi_{t}((0,0, \cdots),(0,0, \cdots))=(0,0, \cdots)$, for $0 \leqq t \leqq 1$,

(5) for each integer $n \geqq 1$ there is an $\varepsilon, 0<\varepsilon<1$, such that $p_{i} \circ p_{1}=$ $p_{i} \circ \varphi_{t}$, for $0 \leqq t \leqq \varepsilon$ and $1 \leqq i \leqq n$,

(6) for each integer $n \geqq 1$ there is an $\varepsilon, 0<\varepsilon<1$, such that $p_{i} \circ p_{2}=$ $p_{i} \circ \varphi_{t}$, for $1-\varepsilon \leqq t \leqq 1$ and $1 \leqq i \leqq n$.

Proof. Define $\varphi_{1 / 2}: F^{\omega} \times F^{\omega} \rightarrow F^{\omega}$ by

$$
\begin{aligned}
p_{i} \circ \varphi_{1 / 2} & =p_{(i+1) / 2} \circ p_{1}, & & \text { for } i \text { odd, } \\
& =p_{i / 2} \circ p_{2}, & & \text { for } i \text { even. }
\end{aligned}
$$

For all integers $n \geqq 2$ define $\varphi_{1 /(n+1)}: F^{\omega} \times F^{\omega} \rightarrow F^{\omega}$ by

$$
\begin{aligned}
& p_{i} \circ \varphi_{1 /(n+1)}=p_{i} \circ p_{1}, \quad \text { for } 1 \leqq i \leqq n, \\
& =p_{i-n} \circ p_{2}, \text { for } n+1 \leqq i \leqq 2 n \text {, } \\
& =p_{i} \circ \varphi_{1 / 2}, \text { for } 2 n+1 \leqq i \text {. }
\end{aligned}
$$

Similarly for all integers $n \geqq 2$ define $\varphi_{n /(n+1)}: F^{\omega} \times F^{\omega} \rightarrow F^{\omega}$ by

$$
\begin{aligned}
p_{i} \circ \varphi_{n /(n+1)} & =p_{i} \circ p_{2}, & & \text { for } 1 \leqq i \leqq n, \\
& =p_{i-n} \circ p_{1}, & & \text { for } n+1 \leqq i \leqq 2 n, \\
& =p_{i} \circ \varphi_{1 / 2}, & & \text { for } 2 n+1 \leqq i .
\end{aligned}
$$

This defines $\varphi$ on the set $\bigcup\left\{F^{\omega} \times F^{\omega} \times\{t\} \mid t=1 /(n+1)\right.$ or $\left.t=n /(n+1)\right\}$. To extend $\varphi$ to all of $F^{\omega} \times F^{\omega} \times I$ put $\varphi_{0}=p_{1}, \varphi_{1}=p_{2}$, and use Lemma 2.1 to extend $\varphi$ to the entire interval joining any two consecutive points of $\{1 /(n+1) \mid n \geqq 1\} \cup\{n /(n+1) \mid n \geqq 1\}$.

We now state and prove the main result of this section. It will be needed in the proof of Theorem 1 .

LEMMA 2.3. Let $X$ be a topological space, $\alpha: X \rightarrow I$ be a map, and let $F$ be a TVS. If $f$ is an $X$-preserving homeomorphism of $X \times F^{\omega}$ onto itself, then 
there is an $X$-preserving homeomorphism $f_{\alpha}$ of $X \times F^{\omega}$ onto itself such that $\left(f_{\alpha}\right)_{x}=f_{x}$, for all $x \in \alpha^{-1}(0)$, and $\left(f_{\alpha}\right)_{x}=\mathrm{id}$, for all $x \in \alpha^{-1}(1)$.

Proof. Let $i: X \times F^{\omega} \rightarrow X \times F^{\omega} \times I$ be defined by $i(x, a)=(x, a, \alpha(x))$, for all $(x, a) \in X \times F^{\omega}$. Then we define $f_{\alpha}$ to be the function which makes the following diagram commute:

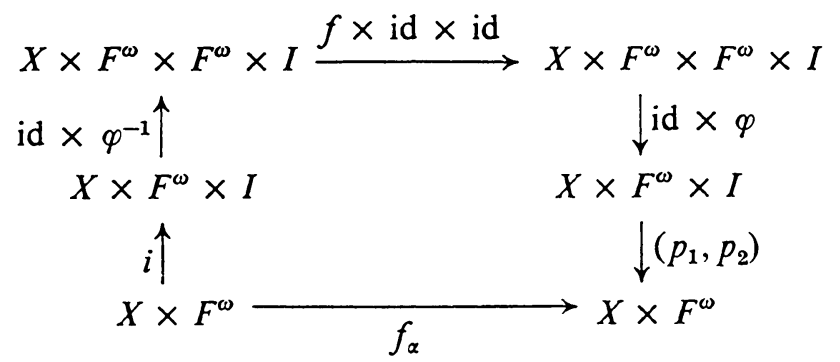

Here $\varphi$ is the function of Lemma 2.2 and $\left(p_{1}, p_{2}\right)$ is projection. To avoid ambiguity we remark that $(\mathrm{id} \times \varphi) \circ(f \times \mathrm{id} \times \mathrm{id}) \circ\left(\mathrm{id} \times \varphi^{-1}\right)(x, a, 0)=$ $(f(x, a), 0)$ and $(\mathrm{id} \times \varphi) \circ(f \times \mathrm{id} \times \mathrm{id}) \circ\left(\mathrm{id} \times \varphi^{-1}\right)(x, a, 1)=(x, a, 1)$, for all $(x, a) \in X \times F^{\omega}$. It can be routinely verified that $f_{\alpha}$ fulfills our requirements.

3. Proof of Theorem 1. We are given a locally-trivial bundle $\xi=$ $(E, p, B)$ with fiber $F^{\omega}$, where $F$ is a TVS. That is $p: E \rightarrow B$ is a map and for each $b \in B$ there is an open set $U \subset B$ containing $b$ and a homeomorphism $h_{U}$ of $U \times F^{\omega}$ onto $p^{-1}(U)$ such that $p \circ h_{U}=p_{1}$. We want to prove that $\xi$ is trivial, i.e. construct a homeomorphism $h$ of $B \times F^{\omega}$ onto $E$ such that $p \circ h=p_{1}$.

Since $B$ is paracompact there exists a locally-finite open cover $\left\{U_{\sigma} \mid \sigma \in \Sigma\right\}$ of $B$ such that for each $\sigma \in \Sigma$ there exists a homeomorphism $h_{\sigma}$ of $U_{\sigma} \times F^{\omega}$ onto $p^{-1}\left(U_{\sigma}\right)$ such that $p \circ h_{\sigma}=p_{1}$. Choose a closed cover $\left\{C_{\sigma} \mid \sigma \in \Sigma\right\}$ of $B$ such that $C_{\sigma} \subset U_{\sigma}$, for all $\sigma \in \Sigma$. Let $\mathscr{G}$ be the set of all pairs $(\Gamma, h(\Gamma))$, where $\Gamma \subset \Sigma$ and $h(\Gamma)$ is a homeomorphism of $\left(\bigcup\left\{C_{\gamma} \mid \gamma \in \Gamma\right\}\right) \times F^{\omega}$ onto $p^{-1}\left(\bigcup\left\{C_{\gamma} \mid \gamma \in \Gamma\right\}\right)$ satisfying $p \circ h(\Gamma)=p_{1}$. We can partially order $\mathscr{G}$ by defining $\left(\Gamma_{1}, h\left(\Gamma_{1}\right)\right) \leqq\left(\Gamma_{2}, h\left(\Gamma_{2}\right)\right)$ if and only if $\Gamma_{1} \subset \Gamma_{2}$ and $h\left(\Gamma_{2}\right)$ agrees with $h\left(\Gamma_{1}\right)$ on

$$
\left[\left(\bigcup\left\{C_{\gamma} \mid \gamma \in \Gamma_{1}\right\}\right) \backslash\left(\bigcup\left\{U_{\gamma}\left|\gamma \in \Gamma_{2}\right| \Gamma_{1}\right\}\right)\right] \times F^{\omega} .
$$

Let $\mathscr{H}$ be any chain in $\mathscr{G}$. We will show that $\mathscr{H}$ has an upper bound.

Note that $(\Gamma, h(\Gamma)) \leftrightarrow \Gamma$ sets up a bijection between $\mathscr{H}$ and a collection $\mathscr{D}$ of subsets of $\Sigma$. Let $\Gamma^{*}=\bigcup\{\Gamma \mid \Gamma \in \mathscr{D}\}$ and for each $\Gamma \in \mathscr{D} \cup\left\{\Gamma^{*}\right\}$ let $C(\Gamma)=\bigcup\left\{C_{y} \mid \gamma \in \Gamma\right\}$. For any element $x \in C\left(\Gamma^{*}\right)$ we can choose an element $\Gamma_{x} \in \mathscr{D}$ such that $x \in U_{x} \subset C\left(\Gamma_{x}\right)$, where $U_{x}$ is relatively open in 
$C\left(\Gamma^{*}\right)$ and $U_{x} \cap U_{\sigma}=\varnothing$, for all $\sigma \in \Gamma^{*} \mid \Gamma_{x}$. Then we define $h\left(\Gamma^{*}\right) \mid U_{x} \times F^{\omega}=$ $h\left(\Gamma_{x}\right) \mid U_{x} \times F^{\omega}$. In this manner we obtain a function $h\left(\Gamma^{*}\right): C\left(\Gamma^{*}\right) \times F^{\omega} \rightarrow$ $p^{-1}\left(C\left(\Gamma^{*}\right)\right)$. It can easily be checked that $h\left(\Gamma^{*}\right)$ is an onto homeomorphism and $p \circ h\left(\Gamma^{*}\right)=p_{1}$. Moreover $\left(\Gamma^{*}, h\left(\Gamma^{*}\right)\right)$ is an upper bound for $\mathscr{H}$.

Using Zorn's lemma it follows that $\mathscr{G}$ has a maximal element $(\Gamma, h(\Gamma))$. We will be done if we can prove that $\Gamma=\Sigma$. Thus assume that there is some element $\sigma \in \Sigma \mid \Gamma$. We will obtain a contradiction to the fact that $(\Gamma, h(\Gamma))$ is maximal. Let $C=\bigcup\left\{C_{\gamma} \mid \gamma \in \Gamma\right\}$ and put $h=(h(\Gamma))^{-1} \circ h_{\sigma} \mid\left(C \cap U_{\sigma}\right) \times F^{\omega}$, which is a $\left(C \cap U_{\sigma}\right)$-preserving homeomorphism of $\left(C \cap U_{\sigma}\right) \times F^{\omega}$ onto itself. Let $\tilde{\alpha}: C \rightarrow I$ be a map such that $C \cap C_{\sigma} \subset \tilde{\alpha}^{-1}(0)$ and $K \subset C \cap U_{\sigma}$, where $K$ is the closure of $\tilde{\alpha}^{-1}([0,1))$ in $C$. Then put $\alpha=\tilde{\alpha} \mid C \cap U_{\sigma}$. Using the notation of Lemma 2.3 (with $X=C \cap U_{\sigma}$ ) consider the homeomorphism $h_{\alpha}$ of $\left(C \cap U_{\sigma}\right) \times F^{\omega}$ onto itself. It follows that $h_{\alpha}\left|\left(C \cap C_{\sigma}\right) \times F^{\omega}=h\right|\left(C \cap C_{\sigma}\right) \times$ $F^{\omega}$ and $h_{\alpha} \mid\left(\left(C \cap U_{\sigma}\right) \backslash K\right) \times F^{\omega}=\mathrm{id}$. Then $h(\Gamma) \circ h_{\alpha}:\left(C \cap U_{\sigma}\right) \times F^{\omega} \rightarrow p^{-1}\left(C \cap U_{\sigma}\right)$ is an onto homeomorphism which satisfies $h(\Gamma) \circ h_{\alpha} \mid\left(C \cap C_{\sigma}\right) \times F^{\omega}=$ $h_{\sigma} \mid\left(C \cap C_{\sigma}\right) \times F^{\omega}$ and $h(\Gamma) \circ h_{\alpha}\left|\left(\left(C \cap U_{\sigma}\right) \backslash K\right) \times F^{\omega}=h(\Gamma)\right|\left(\left(C \cap U_{\sigma}\right) \backslash K\right) \times F^{\omega}$. This means that we can define $g:\left(C \cup C_{\sigma}\right) \times F^{\omega} \rightarrow p^{-1}\left(C \cup C_{\sigma}\right)$ as follows:

$$
\begin{aligned}
g(x, a) & =h(\Gamma)(x, a), & & \text { for }(x, a) \in(C \backslash K) \times F^{\omega}, \\
& =h(\Gamma) \circ h_{\alpha}(x, a), & & \text { for }(x, a) \in\left(C \cap U_{\sigma}\right) \times F^{\omega}, \\
& =h_{\sigma}(x, a), & & \text { for }(x, a) \in C_{\sigma} \times F^{\omega} .
\end{aligned}
$$

It is clear that $(\Gamma \cup\{\sigma\}, g) \in \mathscr{G}$ and $(\Gamma, h(\Gamma))<(\Gamma \cup\{\sigma\}, g)$, contradicting the maximality of $(\Gamma, h(\Gamma))$.

4. Lemmas for Theorem 2. We will first establish a version of Lemma 2.3 where $f: X \times F^{\omega} \rightarrow X \times F^{\omega}$ is an $X$-preserving open embedding (i.e. a homeomorphism into), rather than a homeomorphism onto. At first one might think that such a result would follow directly from the proof of Lemma 2.3, but it will not work. Indeed if $f$ is an open embedding in the proof of Lemma 2.3 , then $f_{\alpha}$ does not necessarily have to be open. The problem arises in the upper part of the diagram used there. Let us call $f^{*}$ the map which makes the following diagram commute:

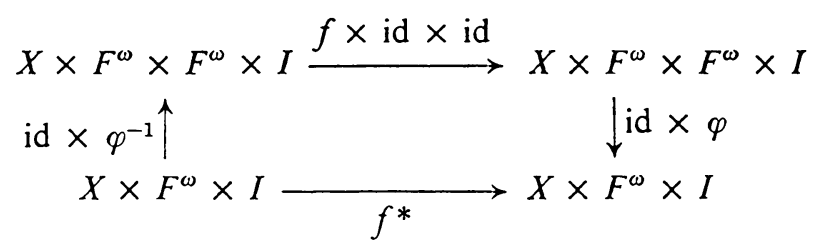

If $f$ is a homeomorphism onto, then so is $f^{*}$. However if $f$ is an open embedding, then $f^{*}$ is an embedding and $f^{*} \mid X \times F^{\omega} \times[0,1)$ is an open embedding, but $f^{*}$ might fail to be open at points of $X \times F^{\omega} \times\{1\}$. Roughly 
the idea is to use $f^{*} \mid X \times F^{\omega} \times\left[0, \frac{1}{2}\right]$ to move "halfway" from $\alpha^{-1}(0)$ to $\alpha^{-1}(1)$. Then we move the rest of the way to $\alpha^{-1}(1)$ by using a modification of Alexander's trick.

LEMMA 4.1. Let $X$ be a topological space, $\alpha: X \rightarrow I$ be a map, and let $F$ be a metric TVS. If $f: X \times F^{\omega} \rightarrow X \times F^{\omega}$ is an $X$-preserving open embedding which satisfies $f \mid X \times\{(0,0, \cdots)\}=\mathrm{id}$, then there is an $X$-preserving open embedding $f_{\alpha}: X \times F^{\omega} \rightarrow X \times F^{\omega}$ such that $f_{x} \mid X \times\{(0,0, \cdots)\}=\mathrm{id},\left(f_{\alpha}\right)_{x}=f_{x}$, for all $x \in \alpha^{-1}(0)$, and $\left(f_{\alpha}\right)_{x}=\mathrm{id}{ }_{X}$, for all $x \in \alpha^{-1}(1)$.

Proof. Let $X_{1}=\alpha^{-1}\left(\left[0, \frac{1}{2}\right]\right)$ and using the notation of Lemma 2.3 let $f_{1}: X_{1} \times F^{\omega} \rightarrow X_{1} \times F^{\omega}$ be the map which makes the following diagram commute:

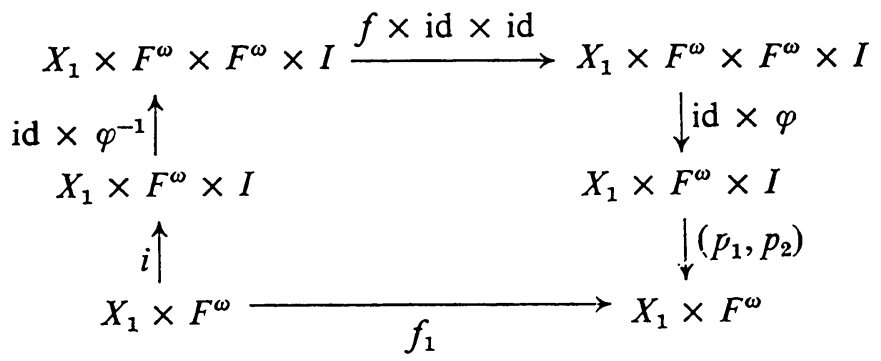

It is easy to check that $f_{1}$ is an open embedding which satisfies $f_{1} \mid X_{1} \times$ $\{(0,0, \cdots)\}=\mathrm{id}, \quad\left(f_{1}\right)_{x}=f_{x}$, for all $x \in \alpha^{-1}(0)$, and $\left(f_{1}\right)_{x}\left(a_{1}, a_{2}, \cdots\right)=$ $\left(a_{1}^{\prime}, a_{2}, a_{3}^{\prime}, a_{4}, \cdots\right)$, where $\left(a_{1}^{\prime}, a_{3}^{\prime}, \cdots\right)=f_{x}\left(a_{1}, a_{3}, \cdots\right)$, for all $x \in \alpha^{-1}\left(\frac{1}{2}\right)$ and $\left(a_{1}, a_{2}, \cdots\right) \in F^{\omega}$. Thus $\left(f_{1}\right)_{x}\left(0, a_{2}, 0, a_{4}, \cdots\right)=\left(0, a_{2}, 0, a_{4}, \cdots\right)$, for all $\left(0, a_{2}, 0, a_{4}, \cdots\right) \in F^{\omega}$ and $x \in \alpha^{-1}\left(\frac{1}{2}\right)$.

Let $X_{2}=\alpha^{-1}\left(\left[\frac{1}{2}, 1\right]\right)$ and let $A=\left\{\left(a_{i}\right) \in F^{\omega} \mid a_{i}=0\right.$ for $i$ odd $\}$. Using Theorem 3.1 of [3] there exists a homeomorphism $u$ of $F^{\omega}$ onto $F^{\omega} \times\left[\frac{1}{2}, 1\right)$ such that $u(A)=F^{\omega} \times\left\{\frac{1}{2}\right\}$. Define $g: X_{2} \times F^{\omega} \rightarrow X_{2} \times F^{\omega}$ to be the open embedding which satisfies $g_{x}\left(a_{1}, a_{2}, \cdots\right)=\left(a_{1}^{\prime}, a_{2}, a_{3}^{\prime}, a_{4}, \cdots\right)$, where $\left(a_{1}^{\prime}, a_{3}^{\prime}, \cdots\right)=$ $f\left(a_{1}, a_{3}, \cdots\right)$, for all $x \in X_{2}$ and $\left(a_{1}, a_{2}, \cdots\right) \in F^{\omega}$. Note that $f_{1} \mid \alpha^{-1}\left(\frac{1}{2}\right) \times$ $F^{\omega}=g \mid \alpha^{-1}\left(\frac{1}{2}\right) \times F^{\omega}$. Also $\tilde{g}=\left(\mathrm{id}_{X_{2}} \times u\right) \circ g \circ\left(\mathrm{id}_{X_{2}} \times u^{-1}\right)$ is an $X_{2}$-preserving open embedding of $X_{2} \times F^{\omega} \times\left[\frac{1}{2}, 1\right)$ into itself which satisfies $\tilde{g}_{x} \mid F^{\omega} \times\left\{\frac{1}{2}\right\}=\mathrm{id}$, for all $x \in X_{2}$.

Using Alexander's trick define $\tilde{g}_{2}: X_{2} \times F^{\omega} \times\left[\frac{1}{2}, 1\right) \rightarrow X_{2} \times F^{\omega} \times\left[\frac{1}{2}, 1\right)$ by the following formula:

$$
\begin{array}{rlrl}
\left(\tilde{g}_{2}\right)_{x}\left(\left(a_{i}\right), t\right) & & \\
& =\left(\left(a_{i}\right), t\right), & & \text { for } \frac{1}{2} \leqq t \leqq \alpha(x), \\
& =w(x) \circ \tilde{g}_{x}\left(\left(a_{i}\right),(t-2 \alpha(x)+1) / 2(1-\alpha(x))\right), & & \text { for } \alpha(x) \leqq t
\end{array}
$$

where $w(x)\left(\left(a_{i}\right), s\right)=\left(\left(a_{i}\right),(2 s-1)(1-\alpha(x))+\alpha(x)\right)$, for $\frac{1}{2} \leqq s \leqq 1$. Note 
that $\left(\tilde{g}_{2}\right)_{x}=\tilde{g}_{x}$, for $x \in \alpha^{-1}\left(\frac{1}{2}\right)$, and $\left(\tilde{g}_{2}\right)_{x}=\mathrm{id}$, for $x \in \alpha^{-1}(1)$. It can easily be checked that $\tilde{g}_{2}$ is an open embedding. Then $f_{2}=\left(\operatorname{id}_{X_{2}} \times u^{-1}\right) \circ \tilde{g}_{2} \circ$ (id $\left.X_{2} \times u\right): X_{2} \times F^{\omega} \rightarrow X_{2} \times F^{\omega}$ is an $X_{2}$-preserving open embedding which satisfies $f_{2}\left|\alpha^{-1}\left(\frac{1}{2}\right) \times F^{\omega}=f_{1}\right| \alpha^{-1}\left(\frac{1}{2}\right) \times F^{\omega}$ and $f_{2} \mid \alpha^{-1}(1) \times F^{\omega}=$ id. Then $f_{\alpha}: X \times$ $F^{\omega} \rightarrow X \times F^{\omega}$ can be defined by $f_{\alpha} \mid X_{1} \times F^{\omega}=f_{1}$ and $f_{\alpha} \mid X_{2} \times F^{\omega}=f_{2}$.

We will also need the following result.

LEMMA 4.2. Let $X$ be a paracompact space and let $F$ be a metric TVS. If $A \subset X$ is closed and $U \subset A \times F^{\omega}$ is a relatively open subset of $A \times F^{\omega}$ which contains $A \times\{(0,0, \cdots)\}$, then there exists an $X$-preserving open embedding $f: X \times F^{\omega} \rightarrow X \times F^{\omega}$ such that $f \mid X \times\{(0,0, \cdots)\}=$ id and $f\left(A \times F^{\omega}\right) \subset U$.

Proof. Let $V$ be an open subset of $X \times F^{\omega}$ which satisfies $V \cap\left(A \times F^{\omega}\right)=$ $U$ and put $W=V \cup\left[(X \backslash A) \times F^{\omega}\right]$, an open set containing $X \times\{(0,0, \cdots)\}$. As an immediate consequence of Lemma 1.2 of [4] and Lemma 2 of [5] there is an $X$-preserving open embedding $f: X \times F^{\omega} \rightarrow X \times F^{\omega}$ such that $f \mid X \times\{(0,0, \cdots)\}=$ id and $f\left(X \times F^{\omega}\right) \subset W$. Clearly $f\left(A \times F^{\omega}\right) \subset U$.

5. Proof of Theorem 2. We are given a microbundle $\xi=(E, i, j, B)$ with fiber $F^{\omega}$, where $F$ is a metric TVS. That is we are given a diagram $B \rightarrow^{i} E \rightarrow^{j} B$, where $B$ is the base space, $E$ is the total space, and $i, j$ are maps such that $j \circ i=\mathrm{id}_{B}$ and the following local triviality condition is satisfied: for each $b \in B$ there exists an open neighborhood $U$ of $b$ and an open embedding $h_{U}: U \times F^{\omega} \rightarrow E$ such that the following diagram commutes:

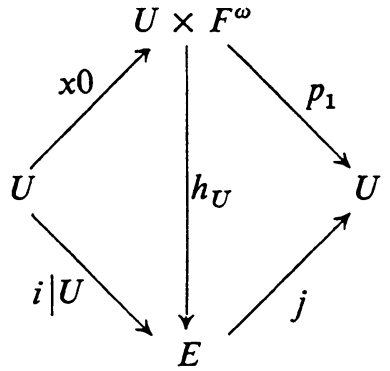

Here $x 0$ denotes the injection $u \rightarrow(u,(0,0, \cdots))$. In the sequel we will write this diagram as $h_{U} \circ(x 0)=i \mid U$ and $j \circ h_{U}=p_{1}$. What we need to do is construct an open embedding $h: B \times F^{\omega} \rightarrow E$ such that $h \circ(x 0)=i$ and $j \circ h=p_{1}$.

The proof now proceeds in a manner similar to the proof of Theorem 1. Zorn's lemma is used in an analogous fashion and we will only prove a result which is analogous to the last part of the proof of Theorem 1 . Thus 
let $C \subset B$ be closed and assume that there exists an embedding $h_{C}: C \times$ $F^{\omega} \rightarrow E$ such that $h_{C}\left(C \times F^{\omega}\right)$ is a relatively open subset of $j^{-1}(C), h_{C}$ 。 $(x 0)=i \mid C$, and $j \circ h_{C}=p_{1}$. Let $A \subset U \subset B$, where $A$ is closed and $U$ is open. Assume that there exists an open embedding $h_{U}: U \times F^{\omega} \rightarrow E$ such that $h_{U} \circ(x 0)=i \mid U$ and $j \circ h_{U}=p_{1}$. What we want to do is construct an embedding $h^{*}:(A \cup C) \times F^{\omega} \rightarrow E$ such that $h^{*}\left((A \cup C) \times F^{\omega}\right)$ is a relatively open subset of $j^{-1}(A \cup C), h^{*} \circ(x 0)=i \mid A \cup C, j \circ h^{*}=p_{1}$, and $h^{*}$ agrees with $h_{C}$ on $(C \backslash U) \times F^{\omega}$.

Using Lemma 4.2 we can assume that $h_{U}\left(U \times F^{\omega}\right) \cap j^{-1}(C) \subset h_{C}\left(C \times F^{\omega}\right)$. Thus (as in the proof of Theorem 1) let $h=h_{C}^{-1} \circ h_{U} \mid(C \cap U) \times F^{\omega}$, which is a $(C \cap U)$-preserving open embedding of $(C \cap U) \times F^{\omega}$ into itself such that $h \mid(C \cap U) \times\{(0,0, \cdots)\}=$ id. Now we use Lemma 4.1 (as Lemma 2.3 was analogously used in the proof of Theorem 1) to obtain a $(C \cap U)$-preserving open embedding $h_{\alpha}$ of $(C \cap U) \times F^{\omega}$ into itself such that $h_{\alpha} \mid(C \cap U) \times$ $\{(0,0, \cdots)\}=$ id,$h_{\alpha}\left|(C \cap A) \times F^{\omega}=h\right|(C \cap A) \times F^{\omega}$, and $h_{\alpha} \mid((C \cap U) \mid K) \times$ $F^{\omega}=\mathrm{id}$, where (as in the proof of Theorem 1) $K$ is the closure of $\tilde{\alpha}([0,1)$ ) in $C$. Then $h_{\alpha}$ is used in a manner similar to the proof of Theorem 1 to obtain our required $h^{*}$.

\section{REFERENCES}

1. R. D. Anderson, Hilbert space is homeomorphic to the countable infinite product of lines, Bull. Amer. Math. Soc. 72 (1966), 515-519. MR 32 \#8298.

2. C. Bessaga and M. I. Kadec, On topological classification of non-separable Banach spaces (preprint).

3. T. A. Chapman, Deficiency in infinite-dimensional manifolds, General Topology and Appl. 1 (1971), 263-272.

4. D. W. Henderson, Microbundles with infinite-dimensional fibers are trivial, Invent. Math. 11 (1970), 293-303.

5. - Corrections and extensions of two papers about infinite-dimensional manifolds, General Topology and Appl. 1 (1971), 321-327.

6. - Stable classification of infinite-dimensional manifolds by homotopy type Invent. Math. 12 (1971), 48-56.

7. J. E. West, Fixed-point sets of transformation groups on infinite-product spaces, Proc. Amer. Math. Soc. 21 (1969), 575-582. MR 39 \#945.

8. R. Y. T. Wong, On homeomorphisms of certain infinite dimensional spaces, Trans. Amer. Math. Soc. 128 (1967), 148-154. MR 35 \#4892.

9. - On homeomorphisms of infinite-dimensional bundles. I (preprint).

Department of Mathematics, University of Kentucky, Lexington, Kentucky 40506 